\title{
Isolement et description de la larve de Leptoconops (Leptoconops) irritans Noé, 1905 (Diptera, Ceratopogonidae)
}

\author{
par J. CLASTRIER \\ Institut National de la Santé et de la Recherche médicale \\ Laboratoire d'Ecologie médicale et de Pathologie parasitaire ( $\mathrm{P}^{\mathrm{r}}$ J.-A. Rioux) \\ Faculté de Médecine, F. 34 -Montpellier
}

\begin{abstract}
Résumé
Description de la larve de Leptoconops (L.) irritans, Noé, trouvée dans le sol entre 10 et $20 \mathrm{~cm}$ de profondeur. Quelques indications sont données sur la technique d'isolement suivie.
\end{abstract}

\section{Summary}

A description of the larva of Leptoconops (L.) irritans Noe and some data on the collecting method used are presented. The larva was found at a depth of 10 to $20 \mathrm{~cm}$ below the surface.

Le Laboratoire d'Ecologie médicale de la Faculté de Médecine de Montpellier a détecté et défini, il y a quelques années, le biotope larvaire de Leptoconops (L.) irritans Noé dans le Midi de la France (J.-A. Rioux et coll., 1968) : «sols halomorphes, basiques ( $\mathrm{pH}$ de l'extrait voisin de 8 ), à dominante limoneuse, présentant en période sèche un aspect polygonal (fentes de retrait) », où seuls, les faciès à Salicornia fruticosa et, dans une mesure moindre, à Arthrocnemum glaucum sont productifs. Une station d'observation y a été installée, dans la Baisse aux Aigrettes, afin « d'en étudier les caractères mésologiques et d'en suivre l'évolution annuelle ».

Dans le cadre des activités de cette station, l'isolement de la larve de L. irritans, encore inconnue, a été tenté au cours du mois de juillet 1970, bien que l'année ait été 
peu favorable à cet égard: aucune agressivité de femelles dans la Baisse ; absence à peu près totale d'adultes dans les pièges d'éclosion disposés sur l'ensemble de la station. Seuls, les pièges $19 \mathrm{~A}$ et $19 \mathrm{~B}$ s'étaient montrés productifs : 49 captures d'adultes sur 77 au total, échelonnées entre le 25 juin et le 23 juillet. C'est à proximité de ces pièges que deux tentatives d'isolement ont été faites, avec succès. La larve de $L$. irritans a été trouvée dans le sol, entre 10 et $20 \mathrm{~cm}$ au-dessous de la surface, le 9 juillet (deux larves au $3^{\circ}$ stade) et le 19 juillet (une larve au $4^{\circ}$ stade, une larve au $3^{\circ}$ stade). Après la description de cette larve, quelques détails seront donnés sur la technique d'isolement suivie.

\section{Description de la larve au $4^{\mathrm{e}}$ stade}

LONGUeur : 4,1 mm; diamètre: de 150 à $200 \mu$ (longueur au $3^{e}$ stade : 3,6 à $3,8 \mathrm{~mm})$.

Cette larve est transparente, le corps gras visible sous la forme de grosses masses blanchâtres; le squelette céphalique interne très apparent à travers le tégument. Elle se dépiace rarement, par reptation; un peu plus souvent, l'extrémité céphalique est animée d'un lent mouvement de balancier; seules les mandibules manifestent une inlassable activité.

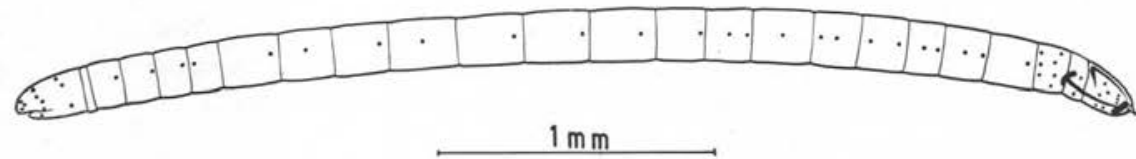

Fig. 1. - Larve de Leptoconops (L.) irritans Noé. Forme générale et répartition des sensilles tégumentaires

Elle est cylindrique, d'un diamètre à peu près constant sur toute sa longueur, et rectiligne, à l'exception de l'extrémité céphalique qui est légèrement coudée vers la face ventrale (fig. 1). Elle compte 23 segments, dont les longueurs respectives sont assez variables d'un spécimen à l'autre, et aussi très irrégulières dans leur répartition chez un même individu. Les plus longs et les plus larges sont cependant toujours situés dans la partie médiane. Les plus courts, dans l'ordre décroissant, sont le prothorax, le mésothorax et les avant-derniers abdominaux (de 2 à 4 suivant les échantillons). A l'exception des deux premiers et du dernier qui seront étudiés plus loin, tous les segments ont la même apparence, sans qu'il soit possible de différencier les segments intercalaires des segments vrais, comme l'avaient déjà noté L. M. Smith et H. Lowe (1948) pour L. (L.) torrens Townsend. Ils sont uniformément clairs, sans relief, sans tache, sans soie ni spicule visibles à l'observation microscopique normale, et sans trace de stigmate respiratoire. Mais ils présentent tous au moins une paire de sensilles semblables à celles qui seront décrites plus loin avec le segment céphalique, et dont la répartition est indiquée sur la figure 1 . 


\section{I. - Extrémité céphalique (fig. 2, 3).}

A) SQuelette interne. Le segment céphalique ne présente pas de capsule hautement sclérifiée comme chez les autres Cératopogonides; en compensation, il possède un squelette interne remarquablement développé, s'étendant jusqu'à l'extrémité antérieure du segment mésothoracique, que l'on peut arbitrairement diviser, pour son étude, en six sections.

1. Squelette ventro-latéral (fig. 2, B, C, C'). Essentiellement constitué par 2 vigoureuses tiges cylindriques $(t v l)$, très fortement sclérifiées, parallèles entre elles, à très légère concavité dorsale, s'étendant depuis la partie antérieure de la tête jusqu'à l'extrémité antérieure du mésothorax. Leur base présente un développement considérable, en forme de fer de pioche, ou de bras d'ancre marine, assurant un véritable ancrage du dispositif, qui paraît devoir s'exercer davantage dans le sens de la traction que dans celui de la pression. Près de son extrémité distale, chacune des tiges présente un épaulement, sur lequel repose le condyle de la plaque externe de l'arc génien (agl).

2. Le squelette dorsal (fig. 2, A, B, B', D) est plus simple: une seule tige dorsomédiane $(t d m)$, s'étendant sur toute la longueur du segment céphalique. Sa partie antérieure, effilée, est en connexion avec l'apodème dorsal $(a f d)$ de chacun des piliers frontaux. Son extrémité postérieure est élargie et forme deux ailerons. Sur chacun des ailerons est soudé un bras dorso-latéral ( $b d l)$, bien sclérifié dans sa partie basale (dorsale), beaucoup moins dans sa partie distale (latérale) dont les limites sont imprécises. Les. deux bras ceinturent la plus grande partie de la base du segment céphalique.

3. Pilier frontal (fig. 2, B, B', D). Schématiquement constitué par un sclérite conique, identifiable sous tous les angles d'observation ( $p f)$, et une masse incolore, striée (striation visible en B), mais souvent cachée ou non reconnaissable, formant un noyau central d'où se détachent trois apodèmes: l'un dorsal (afd) et de direction dorsale, en lame peu sclérifiée (claire sur les préparations, en blanc sur les figures), dont l'extrémité entre en connexion avec la partie antérieure de la tige dorso-médiane déjà vue ; le deuxième fronto-ventral $(a f v)$, plus ou moins cylindrique, peu sslérifié (voir remarque précédente), décrit une large courbe qui le dirige d'abord vers la partie antérieure de la tête, puis le ramène vers la partie médiane, au niveau de la base de la mandibule correspondante ; le troisième $(\mathrm{afm})$, cylindrique et fortement sclérifié (noir sur les préparations et les figures), se dirige presque directement vers la partie médiane de la tête, son extrémité étant plus interne et plus dorsale que celle du précédent.

Ainsi constitué, et indépendamment des attaches musculaires qu'il peut offrir, ce «pilier » unit le squelette dorso-médian au sque'ette ventro-latéral par l'intermédiaire de l'arc génien; il clôt le sommet du segment céphalique ; il établit une ligne transversale de points d'appui pour l'articulation des mandibules et des sclérites qui s'y rattachent.

4. Arc génien (fig. 2, B, B', C, C', D). Cet arc, ainsi nommé en raison de sa situation et par analogie avec le squelette céphalique d'autres Cératopogonides (J. W. H. Lawson, 1951), présente la forme d'une lyre, dont chacune des moitiés paraît composée de trois sclérites. Le plus externe $(a g l)$ en lame épaisse, large, irrégulièrement recti- 
ligne, massif en vue latérale, beaucoup plus délié en vue ventrale. Par son extrémité antérieure, il entre en connexion avec le pilier frontal, au niveau de son élément central conique $(p f)$; il porte d'autre part, dans sa partie moyenne, un vigoureux condyle déjà cité, qui s'articule avec l'épaulement de la tige ventro-latérale correspondante.

Le sclérite intermédiaire (ag2), trapézoïdal, mince et peu sclérifié (clair sur les préparations en vue latérale, blanc sur la figure correspondante), double le tiers postérieur de la lame précédente sur sa face interne. Il se dirige vers le squelette pharyngien.

Le sclérite le plus interne (ag3) peut n'être qu'un prolongement du précédent. Il est replié en $\mathrm{S}$ très serré (fig. 2, D) et ne touche pas son homologue du côté opposé. Dans l'espace resté libre est logée la partie la plus antérieure, effilée, du labium.

5. Pièces buccales (fig. 2, sauf A et G). Les mandibules ( $m d$ ) sont de grande taille, fortement convexes; la base bien développée dans le sens dorso-ventral, étroite transversalement. L'extrémité porte trois dents; les deux internes sont implantées dans le corps par une longue et forte racine; la troisième au contraire ne représente qu'un pli, à $90^{\circ}$, d'une plaque du corps sur son bord externe. A la partie postérieure du même bord se voit une puissante apophyse triangulaire, extrêmement saillante, dirigée postérieurement et vers la partie médiane de la mandibule (ae). Le bord interne subit un grand développement dans le sens sagittal et se prolonge postérieurement, bien au-delà de la base, par une autre apophyse terminée en bouton (ai). Sur la larve au troisième stade (fig. 2, F), l'apophyse externe est dirigée antérieurement et le développement du bord interne est encore plus marqué. Ces mandibules fonctionnent verticalement.

Dans la partie médiane de la face se voient deux formations lamellaires longues, étroites et minces, que nous assimilons aux prémandibules ( $p m d)$. A leur naissance, dans un plan dorsal à celui des mandibules (fig. 2, B'), elles sont légèrement écartées l'une de l'autre et se dirigent vers le sommet de la tête, mais très rapidement elles se rapprochent et se courbent, pour se diriger vers la partie ventrale de la bouche. A leur extrémité, elles sont doublées par une autre lame, très courte, recourbée en sens inverse de la précédente.

Dorsalement à la base de la mandibule se trouve un sclérite, innominé (sci), dont la base s'allonge sur la partie interne et supérieure de la première pièce de l'arc génien, qui décrit ensuite, tout en s'amincissant, une large courbe transversale audevant de l'apodème fronto-ventral, et dont l'extrémité, barrée en $\mathrm{T}$ par deux tiges

FIg. 2. - Extrémité céphalique de la larve de Leptoconops (L.) irritans Noé en vues dorsale (A, $\left.\mathbf{A}^{\prime}\right)$, latérale $\left(B, B^{\prime}\right)$, ventrale $\left(C, C^{\prime}\right)$ et frontale (D). E : mandibule droite vue par sa face externe et sclérite innominé. F: mandibule gauche d'une larve au troisième stade vue par sa face interne et sclérite innominé. G : squelette pharyngien en vue frontale. $a e, a i$ : apophyses externe et interne de la mandibule; an: antenne; afd, afm, afv : apodèmes fronto-dorsal, f-médian, f-ventral, du pilier frontal; agl, ag2, ag3: $1^{\text {re }}, 2^{\mathrm{e}}, 3^{\mathrm{e}}$ plaques de l'arc génien ; bdl : bras dorso-latéral; épi : épipharynx ; hypo: hypopharynx; lbi: labium; lbr: labre; $m d:$ mandibule ; $m t:$ mentum ; pf: sclérite central du pilier frontal; pmd: prémandibules; sci : sclérite innominé ; $t d m$ : tige dorso-médiane ; $t v l$ : tige ventro-latérale 

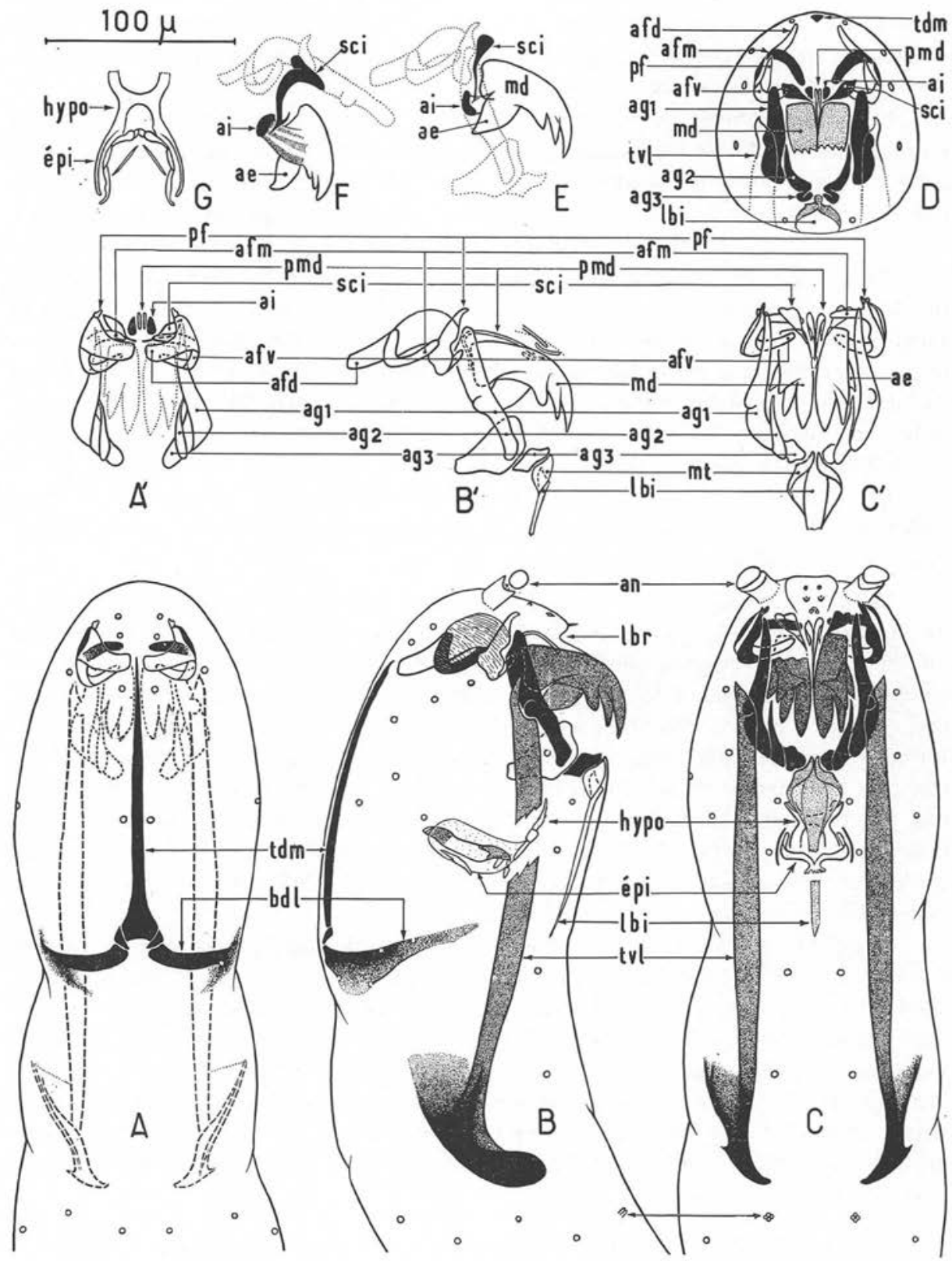
filiformes, vient se p'acer entre la base de la mandibule et le bouton de son apophyse interne. On ne voit pas très bien, dans ces conditions, où s'articule réellement la mandibule, et on peut penser que le sclérite innominé et les prémandibules possèdent une certaine amplitude de mouvement.

Les prémandibules, le bouton mandibulaire interne, le sclérite innominé, sont parfaitement visibles et identifiables en vues frontale et dorsale : les premières $(\mathrm{pmd})$ dans la partie tout à fait médiane, le deuxième (ai) comme accolé à la face externe des précédentes, le troisième (sci) très latéral, longuement piriforme, la «queue » décrivant une petite courbe qui semble rejoindre et prolonger celle de son homologue.

Nous avons déjà vu que dans l'espace resté libre entre les deux pièces internes de l'arc génien (ag3), venait se loger la partie la plus antérieure du labium. Il s'agit de deux petites plaques sclérifiées, soudées, pouvant être assimilées au mentum $(m t)$, auxquelles fait suite le labium proprement dit (lbi). Celui-ci globuleux à la base ; en lame étroite, médiane, mince, peu sclérifiée, à limites imprécises, ailleurs. Son extrémité, mal définie, se situe approximativement au même niveau que les bras dorso-latéraux du squelette dorsal.

Le petit nombre de larves dont nous disposions ne nous a pas permis de multiplier les dissections du squelette de la face, et nous n'avons observé aucune formation susceptible d'être assimilée à une maxille.

6. Squelette pharyngien (fig. 2, B, C, G). Il comprend un hypopharynx et un épipharynx. L'hypopharynx (hypo) est représenté par une lame sans relief, dessinant un $\mathrm{X}$ dont les branches antérieures, largement séparées, sont probablement en connexion plus ou moins lointaine avec le prolongement postérieur du $2^{\circ}$ sclérite de l'arc génien. Chacune des branches postérieures au contraire est prolongée par une formation membraneuse peu sclérifiée, dirigée vers la face dorsale, qui entoure de très près l'épipharynx en le débordant largement sur ses deux faces, mais qui n'atteint pas son extrémité. Ventralement, cette formation apparaît comme une petite lame courbe placée de part et d'autre de l'épipharynx, dont il n'est pas possible de préciser les limites ; latéralement, elle est pratiquement invisible. C'est seulement en vue frontale qu'elle se voit avec une grande netteté.

L'épipharynx (épi) forme un anneau ouvert dorsalement, disposé à $115^{\circ}$ environ sur le plan de l'hypopharynx. Les deux branches qui le forment sont soudées à la base, coudées à angle droit et terminées par trois larges dents largement séparées, dont les deux latérales sont elles-mêmes subdivisées en deux dents secondaires, arrondies, mousses, et la dent centrale en trois dents semblables aux précédentes. Ces branches, bien que régulièrement arrondies, dessinent une petite courbure à convexité postérieure; elles sont légèrement recourbées vers l'extérieur à leur extrémité. Postérieurement à l'anneau et dans sa partie médiane, se voient deux petites sclérifications linéaires disposées en V.

B) Formations sensorielles. Comme sur le reste de la larve, le tégument céphalique est nu; on n'y voit pas de soie ni de spicule; on n'y voit pas non plus de tache oculaire, la larve est aveugle. Mais elle porte une paire d'antennes et de 
nombreux éléments sensoriels, dont les uns sont disséminés sur l'ensemble du segment, et les autres groupés à la partie antérieure de la tête, sur la face.

Antennes (an). Elles sont situées à l'apex du segment, un peu au-dessous du plan médian, largement séparées l'une de l'autre et dirigées ventralement. Elles sont très courtes, formées de deux articles: le premier en tige cylindrique, le deuxième en bouton, séparé du premier par un large sillon circulaire ; l'un et l'autre peu sclérifiés, légèrement aplatis dans le sens dorso-ventral. Sur la face externe du sillon, cinq petites papilles sont disposées en arc de cercle autour d'une sixième, beaucoup plus grosse (fig. 3, A').

Smith et Lowe (loc. cit.) décrivent, chez L. torrens, un anneau sclérifié faisant partie du squelette interne, à l'intérieur duquel l'antenne peut être totalement invaginée, par l'action des muscles qui s'y attachent. Aucune formation semblable n'est visible sur la larve de $L$. irritans. Nous n'avons pas observé les larves vivantes pendant un temps suffisant pour savoir si les antennes sont rétractiles ou non chez cette espèce.

Eléments sensoriels disséminés (fig. 2, A, B, C, D). Le tégument céphalique présente, comme le reste du corps, de nombreux petits éléments, vraisemblablement sensoriels, bien visibles et parfaitement arrondis, dont le centre paraît très légèrement déprimé, sans aucune soie perceptible dans les conditions de l'observation. Sur la figure 2 ils ont été représentés par des cercles approximativement grossis deux fois.

On les trouve sur la totalité du segment céphalique, où les plus antéríeurs, qui sont aussi les plus nombreux, dessinent une couronne subapicale; sur la face ventrale du prothorax; à l'extrémité antérieure du mésothorax, où ils forment une deuxième couronne. Parmi ces derniers, l'élément ventral le plus proche de la ligne médiane est constitué par l'accolement de quatre petites tiges qui, vues de face, se présentent comme quatre petits cercles disposés en croix. Sur la larve au troisième stade, les sensilles sont moins nombreuses d'une façon générale et la couronne paraît manquer, à l'exception de l'élément cruciforme qui présente les mêmes caractères.

Nous signalerons également ici, bien que leur fonction sensorielle soit très hypothétique, quatre petits points blancs semblables à des bulles, paraissant être en rapports étroits avec le tégument, observés sur le bras dorso-latéral $(b d l)$ de la larve au quatrième stade (deux points semblables seulement chez la larve au troisième stade).

Eléments sensoriels de la face (fig. 3, A, A'). Ils sont disposés en quatre séries linéaires et parallèles: deux séries internes, paramédianes et très proches l'une de l'autre, s'étendant depuis le bord postérieur des antennes jusqu'à la base des mandibules; deux séries latérales, très exactement situées au-dessus de l'extrémité antérieure du premier sclérite de l'arc génien; plus un élément isolé, situé de chaque côté, à mi-chemin entre les deux séries précédentes. Ils ont été désignés par un numéro d'ordre. Sur la figure 3, les éléments ou sensilles 1-6 ont été représentés en vues ventrale en $\mathrm{A}$ et latérale en $\mathrm{A}^{\prime} ;$ le $\mathrm{n}^{\circ} 7$ présente à peu près le même aspect quel que soit l'angle d'observation; les sensilles 8-11 sont représentées en vue ventrale sur la gauche de la figure $\mathrm{A}$, et en vue latérale sur sa droite. 
Les sensilles 1 et 2 sont assez semblables à celles observées sur le reste du tégument, elles paraissent seulement un peu plus développées; 1 se présente comme une courte tige et 2 comme un disque de moitié plus court. Un demi-cône disposé en gouttière, épais, à extrémité mousse, forme le principal de la sensille 3 , dont le centre porte en outre une petite papille. La sensille 4 est impaire et médiane. Elle se présente, vue de face, sous la forme de deux petits cercles réunis par une courbe en anse de panier. Les cercles correspondent à la partie supérieure de deux papilles cylindriques. Sur l'anse prennent naissance deux tiges symétriques, effilées à leur extrémité, très légèrement divergentes l'une de l'autre latéralement, dont la direction
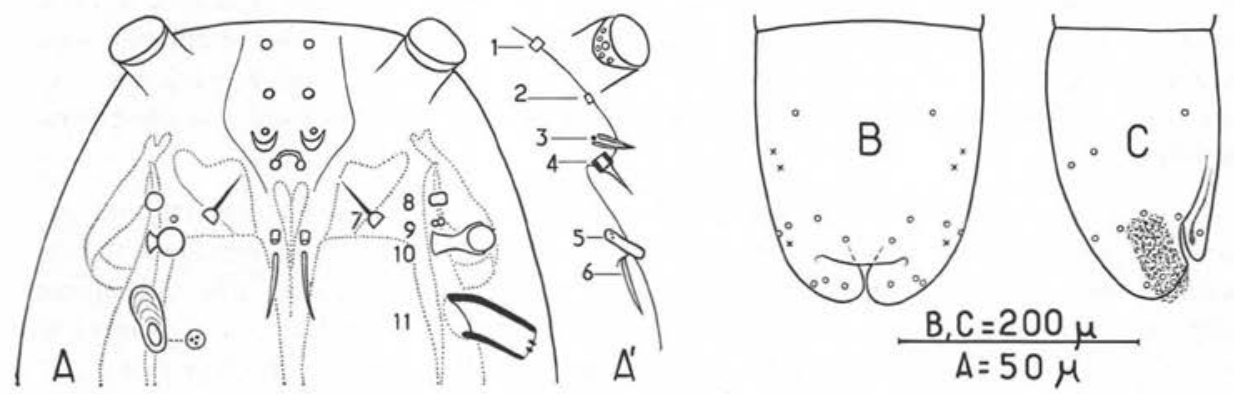

Fig. 3. - Larve de Leptoconops (L.) irritans Noé. Sensilles de la face en vues ventrale (A, sauf 8-11) et latérale $\left(A-8-11, A^{\prime}\right)$, et papilles de l'antenne en vue latérale $\left(A^{\prime}\right)$. Segment anal en vues ventrale (B) et latérale (C) et répartition des sensilles sur ce segment (les sensilles dorsales sont représentées par une croix)

générale est parallèle au tégument. Les sept éléments représentant l'ensemble de ces quatre premières sensilles, sont groupés sur une formation médiane, charnue et saillante du fronto-labre $(l b r)$.

Les deux dernières sensilles de cette série sont nettement détachées des précédentes et placées sur le tégument immédiatement au-dessus du squelette. La $5^{\circ}$ est un long cylindre, à direction antérieure et quelque peu latérale; la $6^{\circ}$ un cône très allongé, appliqué contre le tégument, légèrement concave vers l'extérieur.

D. Keilin (1921), L.-G. Saunders (1924), J.-W. Lawson (1951) ont décrit, chez d'autres Cératopogonides, des formations sensorielles également groupées, par paires, ou impaires, sur le labre. Nous n'avons pu établir aucune homologie avec l'une d'entre elles, sauf peut être entre la «soie sétiforme » de Lawson et la sensille $\mathrm{n}^{\circ} 6$.

L'élément 7 est une épine longue, forte, très acérée, rectiligne, portée par un tubercule bien développé, très difficile à voir.

La sensille 8 est constituée par une courte tige cylindrique, perpendiculaire au plan qui la porte; elle présente quelquefois une légère constriction dans sa partie médiane. La sensille 9 n'est qu'un minuscule champignon, placé au pied de la suivante. L'élément 10 consiste en une longue et forte tige rétrécie en diabolo dans sa 
partie médiane, dont l'extrémité, largement évidée à la manière d'un coquetier, contiendrait une petite sphère transparente. Elle n'est pas tout à fait perpendiculaire au plan qui la porte, mais légèrement inclinée vers la ligne médiane. L'élément 11 est de la même longueur que l'antenne et de moitié moins large. Il s'agit encore d'une tige, aplatie transversalement, dont le bord postérieur est bien plus court que le bord antérieur, ce qui lui donne une position nettement inclinée vers l'arrière. En vue ventrale, son extrémité apparaît elliptique et nue; très rarement, sous certains angles seulement, on la voit arrondie et munie de trois petits points noirs. En vue latérale, seuls les bords antérieur et postérieur sont visibles «en coupe », ainsi que deux des trois points noirs. Par leur taille et leur situation, les éléments 10 et 11 apparaissent avec la plus grande netteté sur les préparations.

Smith et Lowe signalent seulement une paire de soies, en avant des mandibules, chez la larve de $L$. (H.) kerteszi K. et une seule petite soie fourchue, située entre les antennes, chez L. torrens. Sh. M. Dzhafarov (1962) mentionne deux paires de sensilles sur le labre de $L$. (L.) bezzii muganicus, mais aucun de ces auteurs ne fait mention d'autres formations sensorielles. La larve de $L$. irritans serait donc mieux pourvue que ses congénères à cet égard.

II. - Segment anal (fig. 3, B, C). Ce segment est plat sur sa face ventrale, progressivement rétréci latéralement et en hauteur depuis la base jusqu'à son extrémité. Celle-ci porte dorsalement deux lobes bien séparés, arrondis, qui débordent largement, vers l'arrière, le lobe unique et médian de la face ventrale. Entre les trois lobes se trouve l'anus. Plusieurs sensilles tégumentaires sont visibles, disposées comme il est indiqué sur la figure.

\section{Technique d'isolement}

Le sol limono-argileux où vivent les larves de $L$. irritans reste compact et non friable, même après plusieurs heures ou plusieurs jours de contact avec l'eau. Tout au plus se forme-t-il à la surface des mottes une pellicule superficielle, dissociable par une action mécanique légère, mais ne se détachant jamais spontanément, assurant par là l'isolement de la masse sous-jacente. Dans des conditions semblables, Smith et Lowe avaient utilisé un «mouillant»; pour diverses raisons, nous n'avons pu en faire usage. C'est néanmoins la technique sommairement décrite par ces auteurs que nous avons suivie. Elle comprend les trois opérations suivantes: 1) mise en suspension et entraînement de l'échantillon de sol par l'eau ; 2) tamisage ; 3) examen à la loupe binoculaire de la masse retenue sur le tamis.

Pour exécuter les deux premiers temps dans des conditions de travail relativement aisées, il a été nécessaire de confectionner un dispositif très simple. Il s'agit d'un bâti en cornière ajourée du type «meccano », de section carrée de $45 \mathrm{~cm}$ de côté, de $70 \mathrm{~cm}$ de haut, à trois étages (fig. 4). L'étage supérieur (1 sur la figure) est constitué par un vaste entonnoir en zinc, également de section carrée, présentant dans sa partie moyenne un méplat, par lequel il repose sur le bâti. 
L'étage moyen (2 sur la figure) comprend un panneau mobile, reposant latéralement sur deux cornières disposées en glissières, qui permettent de le tirer vers l'avant. Un orifice circulaire de $25 \mathrm{~cm}$ de diamètre permet le passage d'un entonnoir cylindro-conique, retenu sur le panneau par trois équerres soudées. Le tube d'évacuation de cet entonnoir $\mathrm{n}^{\circ} 2$ peut à volonté être partiellement obstrué, par un moyen quelconque, pour rendre son débit insuffisant; en compensation, une série d'ouvertures de $10 \mathrm{~mm}$ de diamètre, jouant le rôle de trop-plein, a été pratiquée sur le pourtour
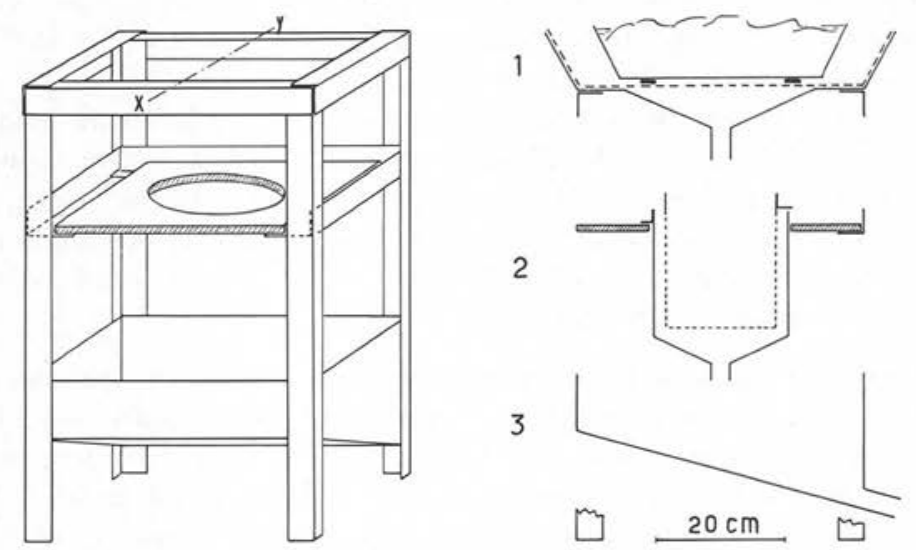

Fig. 4. - Montage pour isolement des larves de Leptoconops irritans. A gauche, le bâti vu par sa face antérieure: 1, étage supérieur nu; 2 , étage moyen et plateau ; 3 , étage inférieur et bac de vidange. A droite, coupe suivant $x y$ des trois étages en place sur le bâti. En 1, de l'extérieur vers l'intérieur: entonnoir de section carrée reposant sur les cornières par son méplat; tamis à larges mailles; les deux barres de fer plat (en noir); cuvette contenant l'échantillon de terre à traiter. En 2: plateau, avec la cornière arrière formant butoir; entonnoir cylindro-conique $n^{\circ} 2$ et un tamis cylindrique en place. En 3: bac de vidange

de la partie cylindrique, à $3 \mathrm{~cm}$ au-dessous du panneau (ces ouvertures non représentées sur la figure). Le ou les tamis cylindriques maintenus à l'intérieur de l'entonnoir par trois équerres soudées se trouvent donc partiellement immergés pendant toute la durée de l'opération, et les larves retenues ne sont pas directement exposées, sur ces tamis, à la chute brutale de la suspension aqueuse de limon (voir plus loin).

L'étage inférieur (3 sur la figure) est un simple bac en zinc, à fond incliné, muni d'une bonde conduisant à l'égout. Un tissu imperméable, amovible et réclinable, peut être disposé intérieurement sur les côtés du bâti, entre les deux étages inférieurs, pour arrêter d'éventuelles éclaboussures.

Le tamisage est effectué en premier lieu par une toile métallique à larges mailles (1 $\mathrm{mm}$ ), épousant les contours de la partie supérieure de l'entonnoir $\mathrm{n}^{\circ} 1$, qui retient les gros débris. Deux autres tamis, cylindriques afin d'augmenter la surface utile et de 
retarder le colmatage, très lâchement emboîtés dans l'entonnoir $\mathrm{n}^{\circ} 2$ comme il a été dit plus haut, assurent la suite des opérations. Ils ont été confectionnés à l'aide des deux toiles immédiatement disponibles, l'une en fil de laiton, l'autre en nylon, respectivement de 300 et de $155 \mu$ d'ouverture de maille.

Les prélèvements de sol sont faits à la bêche, par tranches de $10 \mathrm{~cm}$ d'épaisseur et de $25 \mathrm{~cm}$ de côté environ, depuis la surface jusqu'à $1 \mathrm{~m}$ de profondeur. Chaque tranche est placée dans une cuvette de $35 \times 40 \mathrm{~cm}$, qui est remplie d'eau au laboratoire et abandonnée pendant quelques heures. Elle est ensuite placée dans la partie supérieure de l'entonnoir $\mathrm{n}^{\circ} 1$, sur deux barres de fer plat, parrallèles, prenant appui sur deux côtés opposés du méplat, au-dessus de la toile métallique.

La masse terreuse est alors très largement arrosée, sans interruption, à l'aide d'un tuyau souple d'assez gros diamètre pour assurer un débit abondant sans jet véritable. Une délicate trituration la met lentement en suspension de faible densité. La cuvette peut aussi être agitée comme pour le développement d'une plaque photographique, en faisant déborder une partie du contenu à chacune des oscillations. Pour un prélèvement dont les dimensions ont été données plus haut, l'opération dure de 1 h 30 à 2 heures. Il n'y a aucun intérêt à vouloir aller plus vite, car les tamis se colmatent plus rapidement encore.

La recherche des larves à la loupe stéréoscopique n'a porté que sur le contenu du filtre en nylon. Cette recherche est très longue, les larves ayant sensiblement les mêmes dimensions, la même teinte et la même densité que la masse de radicelles retenues sur le tamis. Fort heureusement, comme on l'a vu, elles ne sont pas totalement immobiles.

Ce montage nécessiterait de nombreux perfectionnements avant de permettre un usage courant. Citons, au nombre de ceux-ci, l'emploi d'un mouillant, de tamis mieux appropriés à cette recherche et leur déplacement sur l'un des côtés du bâti, afin qu'ils restent sous surveillance constante et ne débordent pas lors d'un colmatage. Notons enfin que les tamis $n^{\circ} 60$ et $n^{\circ} 100$ de l'appareil de W. R. Horsfall peuvent être utilisés, mais le dernier est à mailles trop fines pour cet usage et se colmate très rapidement.

Les résultats obtenus sont néanmoins encourageants. Ainsi, pour le secteur $\mathrm{n}^{\circ} 19$ considéré, si l'on rapporte le nombre de larves isolées d'une part, le nombre d'adultes pris dans les pièges d'éclosion d'autre part, à une même unité de surface explorée, on arrive très sensiblement au même ordre de grandeur de 45 à 50 larves au $\mathrm{m}^{2}$.

\section{Bibliographie}

Dzhafarov (Sh. M.), 1962. - Morphology of preimaginal phases of Leptoconops bezzii muganicus Dzhaf. of blood sucking sand-flies (Diptera, Heleidae). Zool. Zl., 41, 241-246.

KeILIN (D.), 1921. - On the Life-history of Dasyhelea obscura, Winnertz (Diptera, Nematocera, Ceratopogonidae), with some Remarks on the Parasites and Hereditary 
Bacterian Symbiont of this Midge. Ann. Mag. nat. Hist., vol. 8 , $9^{\text {e }}$ ser., 576-590, pl. 19 et 20.

LAwson (J. W. H.), 1951. - The Anatomy and Morphology of the early stages of Culicoides nubeculosus Meigen (Diptera, Ceratopogonidae $=$ Heleidae). Trans. R. ent. Soc. London, Vol. 102, Part. 9, 511-570, pl. I.

Rioux (J.-A.), Descous (S.), Corre (J.-J.), Croset (H.) et Tour (S.), 1968. - Ecologie de Leptoconops irritans Noé, 1905 en moyenne Camargue. Localisation et dynamique des biotopes larvaires. Terre et Vie, 458-569.

SAUnders (L. G.), 1924. - On the Life-history and the Anatomy of the early stages of Forcipomyia (Diptera, Nematocera, Ceratopogoninae). Parasit., 16, 164-213, pl. 1012.

Smith (L. M.) et Lows (H.), 1948. - The Black Gnats of California. Hilgardia, 18, 157-183. 